\title{
Plasma Theory of the Many-Electron Atom
}

\author{
Paul H. Levine \\ Department of Physics and Jet Propulsion Laboratory, California Institute of Technology, Pasadena, California \\ AND \\ OLDWIG von Roos \\ Jet Propulsion Laboratory, California Institute of Technology, Pasadena, California
}

(Received July 7, 1961)

\begin{abstract}
A new approach to the many-electron atom, based on the formal equivalence between the Hartree-Fock equations and a quantum-mechanical generalization of the collisionless Boltzmann (Vlasov) equation, is presented. This equivalence casts the problem into the framework of conventional plasma theory, the Vlasov equation being merely replaced by its quantum-mechanical analog. The quantum Vlasov equation permits a straightforward expansion of the quantum-mechanical phase space distribution function in powers of $\hbar$. The first step of this expansion, corresponding physically to a classical correlationless plasma obeying Fermi statistics, leads to the Thomas-Fermi model. Successive steps generate quantum and exchange corrections. The method is applied to the case of the "statistical" correlationless atom (or ion), generalized for the first time to arbitrary temperature and nonzero total orbital angular momentum, with quantum and exchange effects being included to order $\hbar^{2}$.
\end{abstract}

\section{INTRODUCTION}

$\mathrm{T}$ HE Thomas-Fermi statistical model ${ }^{1}$ provides an approximate description of a broad class of spatially inhomogeneous polyelectronic systems, with a degree of success which is surprising in view of its analytical and conceptual simplicity. Attempts to understand this success as well as to improve the model by remedying some of its more obvious shortcomings, have led naturally to investigations of its theoretical foundations.

The first step in this direction was taken by Dirac ${ }^{2}$ who, realizing that the Thomas-Fermi model represents in some sense a classical limit of the Hartree-Fock ${ }^{3}$ theory, rewrote the Hartree-Fock equations in terms of the density matrix, and succeeded thereby in augmenting the Thomas-Fermi model to approximately take account of electron exchange. Subsequent investigations ${ }^{4}$ have generally followed Dirac's lead, in the sense that quasi-classical approximations to the density matrix are studied. In particular, systematic expansions in $\hbar$ have been developed which lead both to exchange and so-called "inhomogeneity" corrections.

These approaches, however, suffer from two drawbacks: (1) Since they are rooted in the Hartree-Fock approximation, correlation effects are excluded; and (2) the conceptual simplicity of the Thomas-Fermi model is destroyed, with the consequence that while the ordinary Thomas-Fermi model can be trivially general-

1 The most recent comprehensive review articles dealing with the Thomas-Fermi model are those of P. Gombas in Handbuch der Physik, edited by S. Flügge (Springer-Verlag, Berlin, 1956), Vol. 36; and N. H. March, in Advances in Physics, edited by N. F. Mott (Taylor and Francis, Ltd., London, 1957), Vol. 6, p. 1. 2 P. A. M. Dirac, Proc. Cambridge Phil. Soc. 26, 376 (1930).

${ }^{3}$ D. Hartree, Proc. Cambridge Phil. Soc. 24, 89 (1928); V. Fock, Phys. Z. Sowjetunion 1, 747 (1932).

${ }^{4}$ W. R. Theis, Z. Physik 142, 503 (1955); A. S. Kompaneets and E. S. Pavlovskii, Soviet Phys.-JETP 4, 328 (1957); D. A. Kirzhnits, Soviet Phys.-JETP 5, 64 (1957); S. Golden, Revs. Modern Phys. 32, 322 (1960). ized, for example, to arbitrary temperatures ${ }^{5}$ or nonzero total orbital angular momenta, ${ }^{6}$ the procedure for systematically obtaining quantum and exchange corrections in these cases is somewhat obscure.

Recently, an attempt to remedy the former of these difficulties by supplanting the density matrix formalism with the more powerful techniques of field theory has been reported. ${ }^{7}$ While such methods hold promise for progress on the correlation question, they unfortunately lead to an aggravation of the second difficulty, since they are based on a formulation of quantum statistical mechanics which is even further conceptually removed from the traditional statistical approach underlying the Thomas-Fermi model than is the density matrix.

In the present paper, therefore, we step in the opposite direction and attempt to establish maximal contact with conventional statistical mechanics by dealing directly with a quantum-mechanical generalization of the phase space density, which turns out, in fact, to be essentially the Fourier transform of the density matrix. This difference, although apparently trivial from a formal standpoint, enables us to cast the problem entirely into the familiar charged-particle statistical mechanics, or "plasma" theory. As a consequence, difficulty (2) vanishes and, although we do not concern ourselves here with (1), i.e., the inclusion of correlation, a close connection between this problem and contemporary difficulties in plasma physics emerges.

Our method is best introduced by briefly outlining the traditional statistical approach to the problem of $N$ identical particles which interact both mutually and with a fixed oppositely charged "nucleus" via their instantaneous Coulomb forces. One begins with the $6 N$ dimensional phase space distribution function,

5 T. Sakai, Proc. Phys. Math. Soc. Japan 24, 254 (1942); R. P. Feynman, N. Metropolis, and E. Teller, Phys. Rev. 75, 1561 (1949).

6 A. M. Sessler and H. M. Foley, Phys. Rev. 96, 366 (1954).

7 G. A. Baraff and S. Borowitz, Phys. Rev. 121, 1704 (1961). 
$F_{N}\left(\mathbf{r}_{1}, \mathbf{r}_{2}, \cdots \mathbf{r}_{N} ; \mathbf{p}_{1}, \mathbf{p}_{2}, \cdots \mathbf{p}_{N} ; t\right)$ whose dynamical (Liouville) equation is obtained from the Newtonian equations of motion. Singlet, doublet, etc., distribution functions are then defined as the integral of $F_{N}$ over $N-1, N-2$, etc., dimensional phase subspaces. The (coupled) dynamical equations for these quantities, the so-called $\mathrm{BBGKY}^{8}$ hierarchy, are obtained by integrating the Liouville equation over appropriate phase subspaces. To proceed further, this system of equations is approximated by truncating the chain at some point. The simplest such approximation is to assume that the doublet distribution is given simply by the product of singlet functions, corresponding to the neglect of all correlations, or "collisions." As is well known, ${ }^{9}$ this procedure leads to the "collisionsless" Boltzmann, or Vlasov $^{10}$ equation for the singlet distribution function, $F_{1}(\mathbf{r}, \mathbf{p}, t)$. The Vlasov equation is satisfied identically by the set $\left\{F_{1}\right\}$ of functionals of the constants of the motion of a single particle in the average field of all the others. The neglect of collisions is then approximately remedied by the ansatz that their effect is to single out that member of $\left\{F_{1}\right\}$ which maximizes the entropy, subject to the constraints on the total system (i.e., total energy, number of particles, angular momentum, etc.). (Tothe extent that only two-body collisions are important, this procedure is validated by the $H$ theorem.) Higher approximations, corresponding to a more exact treatment of correlations, lead to formidable analytical difficulties which are currently being attacked on various fronts. ${ }^{11}$

The question now arises as to how this procedure is to be quantum-mechanically generalized. The simplest generalization is to merely introduce the appropriate quantum statistics into the entropy maximization. This, we find, leads to the Thomas-Fermi model (with Fermi-Amaldi ${ }^{12}$ correction). It is clear, however, that the Vlasov equation must also be quantum-mechanically augmented. This has been done by one of $\mathrm{us}^{13}$ in a previous paper along lines analogous to the derivation of the Vlasov equation sketched above. A quantummechanical phase space distribution function (q.m.d.f.), $\widetilde{F}_{N}\left(\mathbf{r}_{1}, \mathbf{r}_{2}, \cdots \mathbf{r}_{N} ; \mathbf{p}_{1}, \mathbf{p}_{2}, \cdots \mathbf{p}_{N} ; t\right)$, is defined which approaches the classical distribution function as $\hbar \rightarrow 0$. The quantum analog of the Liouville equation for $\widetilde{F}_{N}$ is then obtained from the Schroedinger equation for the $N$-particle wave function, $\psi_{N}$. Decomposition of $F_{N}$ into singlet, doublet, etc., functions leads to the quantum counterpart of the BBGKY chain, which is then truncated, as before, by neglecting correlations. This truncation is equivalent to replacing $\psi_{N}$ by a single

\footnotetext{
${ }^{8}$ A set of equations derived independently by N. N. Bogolubov M. Born, H. S. Green, J. G. Kirkwood, and J. Yvon.

${ }_{9}^{9}$ M. Rosenbluth and N. Rostoker, Phys. Fluids 3, 1 (1960).

${ }_{10}$ A. Vlasov, J. Phys. (USSR) 9, 25 (1945).

11 B. D. Fried and H. W. Wyld, Jr., Phys. Rev. 122, 1 (1961).

12 E. Fermi and E. Amaldi, Mem. accad. Italia 6, 117 (1934).

${ }^{13}$ O. von Roos, Phys. Rev. 119, 1174 (1960).
}

Slater determinant, so that the quantum Vlasov equation thus obtained is formally equivalent to the Hartree-Fock equations. The resulting partial differential equation for the singlet q.m.d.f., $\widetilde{F}$, is introduced in Sec. II. It has the convenient property of permitting a straightforward expansion of $\widetilde{F}$ in powers of $\hbar$, thereby unambiguously generating quantum and exchange corrections from the zeroth-order (ThomasFermi) solution.

We illustrate this method by considering an atom (or ion) at arbitrary temperature, with nonzero total orbital angular momentum. In Sec. III, the ThomasFermi model for this case is derived from the Vlasov equation along the lines sketched above. The quantum and exchange corrections to order $\hbar^{2}$ are then obtained from the quantum Vlasov equation in Sec. IV. In the limit of zero temperature and zero orbital angular momentum, our result agrees with that obtained by others $^{4}$ via the density matrix. In the concluding section, we briefly re-examine the correlation question from the plasma-theoretic point of view developed in this paper.

\section{QUANTUM VLASOV EQUATION}

The quantum Vlasov equation has been derived by one of us in a previous paper, ${ }^{13}$ where it was written in a form facilitating its application to the study of quantum corrected longitudinal plasma oscillations. Since in the present paper we shall be dealing with $N$ electrons (mass $m$, charge $-|e|$ ) in the field of a fixed point nucleus (charge $+Z|e|$ ), rather than the extended homogeneous plasma considered previously, a few trivial modifications are required: (i) the elimination of the action of an electron on itself; (ii) appropriate spin space averaging of the exchange term. ${ }^{14}$ The resulting equation for the singlet q.m.d.f., $\tilde{F}(\mathbf{r}, \mathbf{v}, t)$, can be written as

$$
\begin{aligned}
\left(\frac{\partial}{\partial t}+\mathbf{v} \cdot \nabla_{r}-\frac{1}{m} \nabla_{r} U \cdot \nabla_{v}\right) \tilde{F} \\
=\frac{i \hbar}{2 m}\left\{\nabla_{r}^{2} \widetilde{F}-\frac{1}{m} \sum_{n=0}^{\infty}\left(\frac{-i \hbar}{m}\right)^{n} \frac{2}{(n+2) !}\left(\nabla_{r} \cdot \nabla_{v}\right)^{n+2} U \tilde{F}\right\} \\
\quad+\frac{i e^{2} \hbar}{2 m^{2}} \frac{N-1}{N} \int d^{3} l \exp (i \mathbf{l} \cdot \mathbf{v}) \tilde{f}(\mathbf{r}, \mathbf{l}, t) \\
\quad \times\left\{\frac{1}{l} \tilde{F}\left(\mathbf{r}+\frac{\hbar}{m} \mathbf{l}, \mathbf{v}, t\right)-\frac{1}{2 \pi^{2}} \int \frac{d^{3} v^{\prime}}{\left|\mathbf{v}-\mathbf{v}^{\prime}\right|^{2}}\right. \\
\left.\times \tilde{F}\left(\mathbf{r}+\frac{\hbar}{m} \mathbf{l}, \mathbf{v}^{\prime}, t\right)\right\}
\end{aligned}
$$

\footnotetext{
${ }^{14}$ O. von Roos and J. S. Zmuidzinas, Phys. Rev. 121, 941 (1961).
} 
where

$$
\begin{gathered}
\nabla^{2} U=-4 \pi e^{2} \frac{N-1}{N} \int d^{3} v \widetilde{F}(\mathbf{r}, \mathbf{v}, t), \\
\lim _{r=0} U=-\frac{Z e^{2}}{r}, \quad U \underset{r \rightarrow \infty}{\sim} \frac{(Z-N+1) e^{2}}{r}, \\
\widetilde{F}(\mathbf{r}, \mathbf{v}, t)=(2 \pi)^{-3} \int d^{3} l \tilde{f}(\mathbf{r}, \mathbf{l}, t) \exp (i \mathbf{l} \cdot \mathbf{v}), \\
\left(\nabla_{r} \cdot \nabla_{v}\right)^{n} U \widetilde{F} \equiv \frac{\partial^{n} U}{\partial x_{i} \partial x_{j} \cdots} \frac{\partial^{n} \tilde{F}}{\partial v_{i} \partial v_{j} \cdots},
\end{gathered}
$$

$(i, j, \cdots$ are Cartesian indices running from 1 to 3$)$

$$
\iint d^{3} r d^{3} v \widetilde{F}(\mathbf{r}, \mathbf{v}, t)=N
$$

Gaussian units and the convention that repeated indices are to be summed are used throughout. Information is obtained from $\widetilde{F}$ (a nonobservable) by integration over configuration and velocity space, i.e., if $G(\mathbf{r}, \mathbf{v})$ is any function of $\mathbf{r}$ and $\mathbf{v}$, then

$$
\int G(\mathbf{r}, \mathbf{v}) \widetilde{F}(\mathbf{r}, \mathbf{v}, t) d^{3} r d^{3} v=\langle\psi(t)|\mathcal{G}| \psi(t)\rangle,
$$

where $\mathcal{G}$ is the operator obtained by well-ordering $G\left(\mathbf{r}, \hbar \nabla_{r} / i m\right)$, and $\psi(\mathbf{r}, t)$ is the single-particle HartreeFock wave function for the system. Consequently, the quantum-mechanical expectation value of the electron number density $\rho(\mathbf{r}, t)$ and kinetic energy $K(t)$ of the system, for example, are given by

$$
\rho(\mathbf{r}, t)=\int \widetilde{F}(\mathbf{r}, \mathbf{v}, t) d^{3} v,
$$

and

$$
K(t)=\iint\left[+\frac{1}{2} m v^{2}\right] \widetilde{F}(\mathbf{r}, \mathbf{v}, t) d^{3} r d^{3} v
$$

Since we shall be interested only in the ground state of the system, the time dependence of the above equations will henceforth be suppressed. Consideration of time dependent effects (e.g., collective oscillations) will appear in subsequent papers.

The left-hand side of Eq. (1) is the usual Vlasov operator acting on $\widetilde{F}$. The first term on the right will generate quantum ("inhomogeneity") corrections, while the second term represents the effect of exchange.

\section{Expansion in $\hbar$}

Equations (1)-(3) are conveniently solved by the following iterative expansion in $\hbar$ : First, in Eq. (1),
$U$ is assumed to be given, and the expansion

$$
\widetilde{F}=\sum_{n=0}^{\infty} \hbar^{n} F_{n}
$$

is introduced. $\widetilde{F}(\mathbf{r}+\hbar \mathbf{l} / m, \mathbf{v})$, appearing in the exchange term of (1), is expanded in a Taylor series:

$$
\widetilde{F}(\mathbf{r}+\hbar \mathbf{l} / m, \mathbf{v})=\widetilde{F}(\mathbf{r}, \mathbf{v})+(\hbar \mathbf{l} / m) \cdot \nabla_{r} \widetilde{F}(\mathbf{r}, \mathbf{v})+\cdots
$$

Introducing (10) and (11) into (1) and equating coefficients of similar powers of $\hbar$ leads to the following chain:

0th order

$$
\left[\mathbf{v} \cdot \nabla_{r}-(1 / m) \nabla_{r} U \cdot \nabla_{v}\right] F_{0}=0,
$$

1st order

$$
\begin{aligned}
& {\left[\mathbf{v} \cdot \nabla_{r}-(1 / m) \nabla_{r} U \cdot \nabla_{v}\right] F_{1}} \\
& \quad=(i / 2 m)\left[\nabla_{r}^{2} F_{0}-(1 / m)\left(\nabla_{r} \cdot \nabla_{v}\right)^{2} U F_{0}\right]
\end{aligned}
$$

2nd order

$$
\begin{aligned}
& \left(\mathbf{v} \cdot \nabla_{r}-\frac{1}{m} \nabla_{r} U \cdot \nabla_{v}\right) F_{2} \\
& =\frac{i}{2 m}\left[\nabla_{r}^{2} F_{1}-\frac{1}{m}\left(\nabla_{r} \cdot \nabla_{v}\right)^{2} U F_{1}\right]-\frac{1}{6 m^{3}}\left(\nabla_{r} \cdot \nabla_{v}\right)^{3} U F_{0} \\
& \quad+\frac{2 \pi e^{2}}{m^{3}} \frac{N-1}{N}\left[\nabla_{v} g_{0} \cdot \nabla_{r} F_{0}-\nabla_{v} F_{0} \cdot \nabla_{r} g_{0}\right],
\end{aligned}
$$

where

$$
g_{0}(\mathbf{r}, \mathbf{v})=\int \frac{d^{3} v^{\prime}}{\left|\mathbf{v}-\mathbf{v}^{\prime}\right|^{2}} F_{0}\left(\mathbf{r}, \mathbf{v}^{\prime}\right),
$$

etc. The potential energy $U$ is then rendered selfconsistent by introducing into (2) and (3) the quantity $\widetilde{F}(U ; \mathbf{r}, \mathbf{v})$. (We indicate here explicit dependence of $\widetilde{F}$ on $U$.)

Generally, the series (10) will only be partially summed. Defining the $p$ th approximation to $\widetilde{F}$

$$
\widetilde{F}^{(p)}(U ; \mathbf{r}, \mathbf{v})=\sum_{n=0}^{p} \hbar^{n} F_{n}(U ; \mathbf{r}, \mathbf{v}),
$$

the corresponding approximation $U^{(p)}$ to the potential is then determined from

$$
\begin{aligned}
\nabla^{2} U^{(p)} & =-4 \pi e^{2} \frac{N-1}{N} \int d^{3} v \widetilde{F}^{(p)}\left(U^{(p)} ; \mathbf{r}, \mathbf{v}\right), \\
\lim _{r=0} U^{(p)} & =-\frac{Z e^{2}}{r}, \quad U^{(p)} \underset{r \rightarrow \infty}{\sim} \frac{(Z-N+1) e^{2}}{r},
\end{aligned}
$$

a procedure which guarantees the self-consistency of the potential at every stage of approximation. 


\section{Solution to Second Order}

In this paper, we shall consider only corrections to order $\hbar^{2}$ and hence will require $\widetilde{F}^{(2)}$. Before proceeding to the consideration of special cases, it is useful to point certain simplifications of the relevant equations $[(12)-(14)]$ which can be made in general. First, making use of (12), it is not difficult to show that (13) can be immediately solved to yield

$$
F_{1}=\left(-i / 2 m_{1}\right) \nabla_{r v}^{2} F_{0},
$$

where

$$
\nabla_{r v}^{2} F_{0}=\partial^{2} F_{0} / \partial x_{i} \partial v_{i} \text {. }
$$

Second, again making use of (12), one can establish the identity

$$
\begin{aligned}
& \left(\nabla_{r} \cdot \nabla_{v}\right)^{3} U F_{0}=2 m\left[\nabla_{r}{ }^{2}\left(\nabla_{r v}{ }^{2} F_{0}\right)-(1 / m)\left(\nabla_{r} \cdot \nabla_{v}\right)^{2} U \nabla_{r v}{ }^{2} F_{c}\right] \\
& \quad+m\left[\mathbf{v} \cdot \nabla_{r}\left(\nabla_{r v}{ }^{4} F_{0}\right)-(1 / m) \nabla_{r} U \cdot \nabla_{v}\left(\nabla_{r v}{ }^{4} F_{0}\right)\right], \quad(21)
\end{aligned}
$$

where

$$
\nabla_{r v}{ }^{4} F_{0}=\partial^{4} F_{0} / \partial x_{i} \partial x_{j} \partial v_{i} \partial v_{j} .
$$

Introducing (19) and (21) into (14), there follows

where

$$
F_{2}=-\left(1 / 8 m^{2}\right) \nabla_{r v}{ }^{4} F_{0}+G_{2},
$$

$$
\begin{aligned}
\left(\mathbf{v} \cdot \nabla_{r}-\frac{1}{m} \nabla_{r} U \cdot \nabla_{v}\right) G_{2} & =-\frac{1}{24 m^{3}}\left(\nabla_{r} \cdot \nabla_{v}\right)^{3} U F_{0} \\
& +\frac{2 \pi e^{2}}{m^{3}} \frac{N-1}{N}\left[\nabla_{v} g_{0} \cdot \nabla_{r} F_{0}-\nabla_{v} F_{0} \cdot \nabla_{r} g_{0}\right] .
\end{aligned}
$$

Thus, all that remains is to determine $F_{0}$. Since we are concerned here with the ground state of the system, the $F_{0}$ we seek is that solution of the Vlasov equation (12) corresponding to minimum total energy and maximum entropy.

\section{ATOM OR ION WITH NONZERO ORBITAL ANGULAR MOMENTUM}

As is well known, the most general solution to the Vlasov equation is any functional of the constants of the motion of a particle moving in the potential $U(\mathbf{r})$. We consider an atom or ion with total orbiral angular momentum $J \mathbf{L}$, where $\mathbf{L}$ is a unit vector. It is clear that the potential in this case has rotational symmetry about $\mathbf{L}$, i.e.,

$$
\nabla_{r} U \cdot \mathbf{r} \times \mathbf{L}=0 .
$$

A particle moving in such a potential has only two constants of the motion: the total energy $\frac{1}{2} m v^{2}+U(\mathbf{r})$ and the projection of the orbital angular momentum along $\mathbf{L}$, i.e., $m \mathbf{r} \times \mathbf{v} \cdot \mathbf{L}$. Thus, the most general solution to the Vlasov equation in this case is any functional of the form

$$
F_{0}=F_{0}\left(\frac{1}{2} m v^{2}+U(\mathbf{r}), m \mathbf{r} \times \mathbf{v} \cdot \mathbf{L}\right) .
$$

To minimize the total energy, $\int \mathcal{S}\left[\frac{1}{2} m v^{2}+U(\mathbf{r})\right]$ $\times F_{0}(\mathbf{r}, \mathbf{v}) d^{3} r d^{3} v$, we clearly seek that distribution in velocity space which locally minimizes $(m / 2) \int_{v^{2}} F_{0}(\mathbf{r}, \mathbf{v})$ $\times d^{3} v$ subject to the constraints of fixed spatial density $\int F_{0} d^{3} v$ and fixed local momentum density $\int m \mathbf{v} F_{0} d^{3} v$. It is easily shown that this requirement is met if $F_{0}$ possesses spherical symmetry in velocity space (about some displaced origin), i.e.,

$$
F_{0}=F_{0}\left([\mathbf{v}-\mathbf{d}(\mathbf{r})]^{2}, e(\mathbf{r})\right),
$$

where $\mathbf{d}$ and $e$ are arbitrary functions of $\mathbf{r}$. Combining (26) and (27) leads to a unique form of the minimum energy solution to the Vlasov equation

$$
F_{0}=F_{0}\left(U(\mathbf{r})+\frac{1}{2} m \mathbf{v}^{2}-m \omega \mathbf{r} \times \mathbf{v} \cdot \mathbf{L}\right),
$$

where $\omega$ is a constant. Note that this implies uniform rotation with angular frequency $\omega$. If we transform to the rotating frame, the transformed density function $F_{0}{ }^{\prime}$ is

$$
F_{0}{ }^{\prime}=F_{0}{ }^{\prime}\left(U\left(\mathbf{r}^{\prime}\right)+\frac{1}{2} m v^{2}-\frac{1}{2} m \omega^{2}\left(\mathbf{r}^{\prime} \times \mathbf{L}\right)^{2}\right),
$$

and is therefore a function solely of the energy $\epsilon^{\prime}$, in the rotating frame, where the effective potential energy is

$$
U_{\text {eff }}{ }^{\prime}=U\left(\mathbf{r}^{\prime}\right)-\frac{1}{2} m \omega^{2}\left(\mathbf{r}^{\prime} \times \mathbf{L}\right)^{2} .
$$

The entropy maximization in the rotating frame yields, of course, the Fermi distribution, i.e.,

$$
F_{0}^{\prime}\left(\epsilon^{\prime}\right)=F_{0}\left(\epsilon^{\prime}\right)=2\left(\frac{m}{h}\right)^{3}\left[\exp \left(\frac{\epsilon^{\prime}-\lambda}{k T}\right)+1\right]^{-1} .
$$

The function $F_{0}$ is therefore determined, and we have finally

$$
\begin{aligned}
F_{0}= & 2\left(\frac{m}{h}\right)^{3} \\
& \times\left[\exp \left(\frac{U(\mathbf{r})+\frac{1}{2} m v^{2}-m \omega \mathbf{r} \times \mathbf{v} \cdot \mathbf{L}-\lambda}{k T}\right)+1\right]^{-1} .
\end{aligned}
$$

The Thomas-Fermi (Amaldi) model, generalized to nonzero angular momentum and nonzero temperature, follows from (32), (17), and (18) if $p=0$, i.e., if we use only the lowest order approximation to $\widetilde{F}$. In the limit of zero temperature, this leads to the equations first derived and studied by Sessler and Foley. ${ }^{6}$ For zero angular momentum, we obtain the usual finitetemperature Thomas-Fermi model. ${ }^{5}$

\section{QUANTUM AND EXCHANGE CORRECTIONS}

Using the $F_{0}$ given by (32), we shall now go to second order in and compute $\widetilde{F}^{(2)}$. From (19), (25), and (32) 
there follows immediately

$$
F_{1}=(-i / 2)\left[\mathbf{v} \cdot \nabla_{r} U+m \omega^{2} \mathbf{v} \times \mathbf{L} \cdot \mathbf{L} \times \mathbf{r}\right] F_{0}{ }^{\prime \prime},
$$

where the primes indicate differentiation of $F_{0}$ with respect to its argument, i.e.,

$$
\begin{aligned}
F_{0}{ }^{\prime} \equiv & \left\{\frac{d}{d x} 2\left(\frac{m}{h}\right)^{3}\right. \\
& \left.\times\left[\exp \left(\frac{x-\lambda}{k T}\right)+1\right]^{-1}\right\}_{x=U+\frac{1}{2} m v^{2}-m \omega \mathbf{r} \times \mathbf{v} \cdot \mathbf{L} .}
\end{aligned}
$$

The solution of (23) and (24) for $F_{2}$, while simple and straightforward in the absence of angular momentum, is somewhat involved in the general case and is therefore delegated to the Appendix. The result is

$$
\begin{aligned}
F_{2}= & -\frac{1}{4 m} F_{0}^{\prime \prime} \nabla_{r}^{2} U-\frac{1}{6} F_{0}^{\prime \prime \prime}\left[\mathbf{v} \cdot \nabla_{r}\left(\mathbf{v} \cdot \nabla_{r} U\right)\right. \\
& \left.+\frac{1}{m} \nabla_{r} U \cdot \nabla_{r} U\right]+\frac{\omega}{2} F_{0}^{\prime \prime \prime} \mathbf{v} \cdot \mathbf{L} \times \nabla_{r} U-\frac{1}{8} F_{0}^{\prime \prime \prime \prime} \\
& \times\left\{v \cdot \nabla_{r}\left[U-\frac{1}{2} m \omega^{2}(\mathbf{L} \times \mathbf{r})^{2}\right]\right\}^{2}-\frac{2 \pi e^{2}}{m^{2}} \frac{N-1}{N} F_{0}^{\prime} \\
& \times \int \frac{d^{3} v^{\prime}}{\left|\mathbf{v}-\mathbf{v}^{\prime}\right|^{2}} F_{0}\left(\mathbf{r}, \mathbf{v}^{\prime}\right) .
\end{aligned}
$$

Introducing (32), (34), and (35) into (17) and (18) and performing the angular integrations in velocity space [keeping in mind the symmetry condition (25)] leads after some algebra to the following equation for $U^{(2)}$ :

$$
\begin{aligned}
\nabla^{2} U^{(2)}= & -\frac{4 e^{2}(2 m)^{\frac{3}{2}}}{3 \hbar^{3}} \frac{N-1}{N}\left\{\frac{3}{2} \int_{0}^{\infty} d w w^{\frac{1}{2}} f_{0}\left(w+U^{(2)}-\frac{1}{2} m \omega^{2}(\mathbf{L} \times \mathbf{r})^{2}\right)+\frac{\hbar^{2}}{16 m} \nabla^{2} U^{(2)} \int_{0}^{\infty} d w w^{-\frac{1}{2}} f_{0}\left(w+U^{(2)}-\frac{1}{2} m \omega^{2}(\mathbf{L} \times \mathbf{r})^{2}\right)\right. \\
& +\frac{\hbar^{2}}{32}\left[\frac{1}{m} \nabla U^{(2)} \cdot \nabla U^{(2)}-\omega^{2} \nabla U^{(2)} \cdot \nabla(\mathbf{L} \times \mathbf{r})^{2}-\frac{3}{4} m \omega^{4}\left[\nabla(\mathbf{L} \times \mathbf{r})^{2}\right]^{2}\right] \int_{0}^{\infty} d w w^{-\frac{1}{2}} f_{0}^{\prime \prime}\left(w+U^{(2)}-\frac{1}{2} m \omega^{2}(\mathbf{v} \times \mathbf{L})^{2}\right) \\
& \left.+\frac{3 e^{2}(2 m)^{\frac{1}{2}}}{8 \pi \hbar} \frac{1}{N}\left[\int_{0}^{\infty} d w w^{-\frac{1}{2}} f_{0}\left(w+U^{(2)}-\frac{1}{2} m \omega^{2}(\mathbf{L} \times \mathbf{r})^{2}\right)\right]^{2}\right\}, \quad(36)
\end{aligned}
$$

where

$$
f_{0}(x)=\left[\exp \left(\frac{x-\lambda}{k T}\right)+1\right]^{-1},
$$

and $U^{(2)}$ satisfies the boundary conditions

$$
\begin{gathered}
U^{(2)} \underset{r \rightarrow \infty}{\sim}-(Z-N+1) e^{2} / r \\
\lim _{r=0} U^{(2)}=-Z e^{2} / r .
\end{gathered}
$$

In the limit of zero temperature, the integrals occurring in (36) can be easily performed, yielding

$$
\begin{aligned}
& \nabla^{2} U^{(2)}=-\frac{4 e^{2}}{3 \pi \hbar^{3}} \frac{N-1}{N}\left\{2 m\left[\lambda-U^{(2)}+\frac{1}{2} m \omega^{2}(\mathbf{L} \times \mathbf{r})^{2}\right]\right\}^{\frac{3}{2}}\left\{1-\frac{\hbar^{2}}{16 m} \nabla^{2} U^{(2)}\left[\lambda-U^{(2)}+\frac{1}{2} m \omega^{2}(\mathbf{L} \times \mathbf{r})^{2}\right]^{-2}\right. \\
&-\frac{\hbar^{2}}{64}\left[\lambda-U^{(2)}+\frac{1}{2} m \omega^{2}(\mathbf{L} \times \mathbf{r})^{2}\right]^{-3}\left[\frac{1}{m} \nabla U^{(2)} \cdot \nabla U^{(2)}-\omega^{2} \nabla U^{(2)} \cdot \nabla(\mathbf{L} \times \mathbf{r})^{2}-\frac{3}{4} m \omega^{4}\left[\nabla(\mathbf{L} \times \mathbf{r})^{2}\right]^{2}\right] \\
&\left.+\frac{3 e^{2}(2 m)^{\frac{1}{2}}}{2 \pi \hbar} \frac{1}{N}\left[\lambda-U^{(2)}+\frac{1}{2} m \omega^{2}(\mathbf{L} \times \mathbf{r})^{2}\right]^{-\frac{1}{2}}\right\} .
\end{aligned}
$$

To establish contact with previously published results, ${ }^{4}$ we pass to the limit of zero angular momentum $(\omega \rightarrow 0)$ in $(40)$ and let

$$
U^{(2)}=U_{0}+\hbar^{2} U_{2},
$$

where $U_{0}$ is the solution of the usual Thomas-Fermi (Amaldi) equation :

$$
\nabla^{2} U_{0}=-\frac{4 e^{2}}{3 \pi \hbar^{3}} \frac{N-1}{N}\left[2 m\left(\lambda-U_{0}\right)\right]^{\frac{3}{2}}
$$

Assuming $\hbar^{2} U_{2} \ll U_{0}$ so that only the lowest order terms are kept, (40) becomes

$$
\begin{aligned}
& -\nabla^{2} U_{2}+\frac{4 m e^{2}}{\pi \hbar^{3}} \frac{N-1}{N}\left[2 m\left(\lambda-U_{0}\right)\right]^{\frac{1}{2}} U_{2} \\
& =\frac{8 m^{2} e^{4}}{\pi^{2} \hbar^{6}}\left(\frac{N-1}{N}\right)^{2}\left(\lambda-U_{0}\right)-\frac{m e^{2}}{12 \pi \hbar^{3}} \frac{N-1}{N}\left[2 m\left(\lambda-U_{0}\right)\right]^{-\frac{1}{2}} \\
& \quad \times\left[4 \nabla^{2} U_{0}+\left(\lambda-U_{0}\right)^{-1} \nabla U_{0} \cdot \nabla U_{0}\right],
\end{aligned}
$$


which is the usual quantum and exchange correction to the Thomas-Fermi model, ${ }^{15}$ augmented by the FermiAmaldi $(N-1) / N$ factor. Note that the perturbation procedure used to obtain (43) from (40) destroys the self-consistency of the potential.

Equations (36) and (37) contain two constants which require some discussion. The chemical potential $\lambda$ is essentially determined by the boundary condition (38). The determination of the angular frequency $\omega$ depends on the problem being studied. That is, if the total angular momentum $\mathbf{J}$ is put in as an ad hoc constraint, then $^{16} \omega$ is determined from

$$
\mathbf{J}=\iint d^{3} r d^{3} v m \mathbf{r} \times \mathbf{v} \widetilde{F}^{(2)}(\mathbf{r}, \mathbf{v})
$$

On the other hand, in the spirit of the statistical approach to the atom, one may determine $\omega$ from the model itself, as that which minimizes the total energy. This point will be explored more fully elsewhere.

\section{CORRELATIONS}

The "quantum plasma" approach to the statistical atom presented here, establishes a connection between contemporary problems in plasma physics and the difficult problem of introducing correlations into the Thomas-Fermi model. Correlations are introduced in classical plasma physics by higher order truncations of the BBGKY hierarchy. Thus, for example, instead of decomposing the doublet distribution function into a product of singlet functions, the triplet distribution function is decomposed into products of singlet and doublet functions. This results in a complicated set of coupled equations for the singlet and doublet distributions. ${ }^{9}$ A precisely analogous procedure can be carried through for the q.m.d.f. An expansion of the singlet and doublet q.m.d.f. in powers of $\hbar$ can then be performed, leading to essentially the classical equations in lowest order. Recalling that the Thomas-Fermi "approach" is wholly tantamount to the solution of these lowest order equations, it is clear that to introduce correlations into the Thomas-Fermi model, one must begin by finding the classical doublet distribution function for a spatially inhomogeneous plasma whose singlet function is the Fermi distribution. This problem is as yet unsolved.

\section{APPENDIX. SOLUTION FOR $F_{2}$}

We present here the solution of (23) and (24), where

$$
F_{0}=F_{0}\left(U(\mathbf{r})+\frac{1}{2} m v^{2}-m \omega v \cdot \mathbf{L} \times \mathbf{r}\right) .
$$

Let

$$
F_{2}=F_{2}{ }^{A}+F_{2}{ }^{B}+F_{2}{ }^{C},
$$

\footnotetext{
${ }^{15} \mathrm{See}$, for example, Eq. (4.15) of reference 7.
} ${ }^{16}$ More properly $\omega^{(2)}$, in the sense of the definition (16) where

$$
\begin{aligned}
F_{2}{ }^{A} & =-\frac{1}{8 m^{2}} \nabla_{r v}{ }^{4} F_{0} \\
\left(\mathbf{v} \cdot \nabla_{r}-\frac{1}{m} \nabla_{r} U \cdot \nabla_{v}\right) F_{2}{ }^{B} & =-\frac{1}{24 m^{3}}\left(\nabla_{r} \cdot \nabla_{v}\right)^{3} U F_{0},
\end{aligned}
$$

and

$$
\begin{aligned}
\left(\mathbf{v} \cdot \nabla_{r}-\frac{1}{m} \nabla_{r} U \cdot \nabla_{v}\right) F_{2}{ }^{C} & \\
& =\frac{2 \pi e^{2}}{m^{3}} \frac{N-1}{N}\left(\nabla_{v} g_{0} \cdot \nabla_{r} F_{0}-\nabla_{v} F_{0} \cdot \nabla_{r} g_{0}\right) .
\end{aligned}
$$

In solving these equations, use is continually made of the symmetry condition (25) and related identities like:

$$
\mathbf{v} \cdot \nabla_{r}\left(\mathbf{L} \times \mathbf{r} \cdot \nabla_{r} U\right)=\mathbf{L} \times \mathbf{r} \cdot \nabla_{r}\left(\mathbf{L} \times \mathbf{r} \cdot \nabla_{r} U\right)=0,
$$

etc., to reduce the complexity of the multiple vector and tensor products which formally arise. As space does not permit these manipulations to be exhibited in detail, we present only the skeleton of the calculation.

(A-3) involves only the straightforward evaluation of $\nabla_{r v}{ }^{4} F_{0}$, and we find

$$
\begin{aligned}
F_{2}{ }^{A}= & -(1 / 8 m) F_{0}{ }^{\prime \prime} \nabla^{2} U-\frac{1}{8} F_{0}{ }^{\prime \prime \prime}\left[\mathbf{v} \cdot \nabla_{r}(\mathbf{v} \cdot \nabla U)\right. \\
& \left.+(1 / m)(\nabla U)^{2}\right]+\frac{1}{4} \omega F_{0}{ }^{\prime \prime \prime} \mathbf{v} \cdot \mathbf{L} \times \nabla U \\
& +\frac{1}{16} \omega^{2} F_{0}{ }^{\prime \prime \prime} \nabla_{r}(\mathbf{L} \times \mathbf{r})^{2} \cdot \nabla U+\frac{1}{8} m \omega^{2} F_{0}{ }^{\prime \prime \prime} \\
& \times(\mathbf{v} \times \mathbf{L})^{2}-F_{0}^{\prime \prime \prime \prime}\left\{\mathbf{v} \cdot \nabla_{r}\left[U-\frac{1}{2} m \omega^{2}(\mathbf{L} \times \mathbf{r})^{2}\right]\right\}^{2} \\
& +\left\{\frac{1}{4} m \omega^{2}\left(F_{0}{ }^{\prime \prime}-\omega F_{0}{ }^{\prime \prime \prime} v \cdot \mathbf{L} \times \mathbf{r}\right)\right\} .
\end{aligned}
$$

The last term in curly brackets being a solution of the homogeneous (Vlasov) equation is then deleted since we are only interested in the (inhomogeneous) terms generated directly by the right hand side of (14). Its appearance in (A7) is a formal consequence of the shortcut (21), and care must consequently be taken when evaluating (A3) to delete these spurious terms.

The only real algebraic complexity arises in (A4). Straightforward evaluation of the right-hand side yields.

$$
\begin{aligned}
& -\frac{1}{24 m^{3}}\left(\nabla_{r} \cdot \nabla_{v}\right)^{3} U F_{0} \\
& =-\frac{1}{8 m}\left\{[\mathbf{v}-\omega(\mathbf{L} \times \mathbf{r})] \cdot \nabla_{r}\left[\nabla_{r}^{2} U\right]\right\} F_{0}^{\prime \prime} \\
& \quad-\frac{1}{24}(\mathbf{v}-\omega \mathbf{L} \times \mathbf{r})_{i}(\mathbf{v}-\omega \mathbf{L} \times \mathbf{r})_{j}(\mathbf{v}-\omega \mathbf{L} \times \mathbf{r})_{k} \\
& \times \frac{\partial^{3} U}{\partial x_{i} \partial x_{j} \partial x_{k}} F_{0}^{\prime \prime \prime} .
\end{aligned}
$$

It is not difficult to show that as a consequence of (25),

$\mathbf{L} \times \mathbf{r} \cdot \nabla_{r}\left(\nabla_{r}^{2} U\right)$

$$
=0=(\mathbf{L} \times \mathbf{r})_{i}(\mathbf{L} \times \mathbf{r})_{j}(\mathbf{L} \times \mathbf{r})_{k} \frac{\partial^{3} U}{\partial x_{i} \partial x_{j} \partial x_{k}} .
$$


Consequently, (A8) becomes

$$
\begin{aligned}
& -\frac{1}{24 m^{3}}\left(\nabla_{r} \cdot \nabla_{v}\right)^{3} U F_{0} \\
& =-\frac{1}{8 m}\left[\mathbf{v} \cdot \nabla\left(\nabla^{2} U\right)\right] F_{0}^{\prime \prime}-\frac{1}{24}\left[\mathbf{v} \cdot \nabla_{r}\left(\mathbf{v} \cdot \nabla_{r}(\mathbf{v} \cdot \nabla U)\right)\right] F_{0}^{\prime \prime \prime} \\
& +\frac{1}{8} \omega(\mathbf{L} \times \mathbf{r})_{i} v_{j} v_{k} \frac{\partial^{3} U}{\partial x_{i} \partial x_{j} \partial x_{k}} F_{0}^{\prime \prime \prime}-\frac{1}{8} \omega^{2}(\mathbf{L} \times \mathbf{r})_{i}(\mathbf{L} \times \mathbf{r})_{j} v_{k} \\
& \times \frac{\partial^{3} U}{\partial x_{i} \partial x_{j} \partial x_{k}} F_{0}^{\prime \prime} . \quad(\text { (A10) }
\end{aligned}
$$

The contribution of the first two terms of (A10) to $\mathrm{F}_{2}{ }^{B}$ is immediately found, since it is readily verified that

$$
\begin{gathered}
\left(\mathbf{v} \cdot \nabla_{r}-\frac{1}{m} \nabla_{r} U \cdot \nabla_{v}\right)\left(\frac{-1}{8 m} F_{0}{ }^{\prime \prime} \nabla^{2} U-\frac{1}{24} F_{0}{ }^{\prime \prime \prime}\right. \\
\left.\times\left[\mathbf{v} \cdot \nabla_{r}(\mathbf{v} \cdot \nabla U)+\frac{1}{m}(\nabla U)^{2}\right]\right) \\
=-\frac{1}{8 m}\left[\mathbf{v} \cdot \nabla\left(\nabla^{2} U\right)\right] F_{0}^{\prime \prime}-\frac{1}{24} \\
\times \mathbf{v} \cdot \nabla_{r}\left[\mathbf{v} \cdot \nabla_{r}(\mathbf{v} \cdot \nabla U)\right] F_{0}^{\prime \prime \prime} .
\end{gathered}
$$

The contribution arising from the remaining terms of (A10) proceeds as follows. Using the identity

one finds

$$
\mathbf{v} \cdot \nabla_{r}\left[\mathbf{v} \cdot \nabla_{r}(\mathbf{L} \times \mathbf{v} \cdot \nabla U)\right]=0,
$$

$$
\begin{aligned}
\frac{1}{8} \omega(\mathbf{L} \times \mathbf{r})_{i} v_{j} v_{k} \frac{\partial^{3} U}{\partial x_{i} \partial x_{j} \partial x_{k}} F^{\prime \prime \prime} & \\
& =\frac{1}{4} \omega \mathbf{v} \times \mathbf{L} \cdot \nabla_{r}(\mathbf{v} \cdot \nabla U) F_{0}{ }^{\prime \prime \prime} .
\end{aligned}
$$

This leads to a contribution to $F_{2}{ }^{B}$ of

$$
\frac{1}{4} \omega F_{0}^{\prime \prime \prime} \mathbf{v} \times \mathbf{L} \cdot \nabla U
$$

which can be verified by direct substitution into the left-hand side of (A4).

In a similar fashion, use of the identity

$$
\mathbf{L} \times \mathbf{r} \cdot \nabla_{r}\left(\mathbf{v} \cdot \nabla_{r}(\mathbf{L} \times \mathbf{r} \cdot \nabla U)\right)=0 .
$$

leads, after some algebra, to the relation

$$
\begin{aligned}
& (\mathbf{L} \times \mathbf{r})_{i}(\mathbf{L} \times \mathbf{r})_{j} v_{k} \frac{\partial^{3} U}{\partial x_{i} \partial x_{j} \partial x_{k}} \\
& \quad=\frac{1}{2} \mathbf{v} \cdot \nabla_{r}\left[\nabla_{r}(\mathbf{L} \times \mathbf{r})^{2} \cdot \nabla U\right]-\nabla_{v}(\mathbf{L} \times \mathbf{v})^{2} \cdot \nabla_{r} U .
\end{aligned}
$$

The contribution to $F_{2}{ }^{B}$ arising from the last term of (A10) is immediately apparent and is

$$
-\frac{1}{16} \omega^{2} F_{0}{ }^{\prime \prime \prime}\left[\nabla_{r}(\mathbf{L} \times \mathbf{r})^{2} \cdot \nabla U+2 m(\mathbf{L} \times \mathbf{v})^{2}\right] .
$$

Collecting terms, there follows for $F_{2}{ }^{B}$ :

$$
\begin{aligned}
F_{2}{ }^{B}= & -\frac{1}{8 m} F_{0}^{\prime \prime} \nabla^{2} U-\frac{1}{24} F_{0}{ }^{\prime \prime \prime}\left[\mathbf{v} \cdot \nabla_{r}(\mathbf{v} \cdot \nabla U)+\frac{1}{m}(\nabla U)^{2}\right] \\
& +\frac{1}{4} \omega F_{0}{ }^{\prime \prime \prime} \mathbf{v} \times \mathbf{L} \cdot \nabla U-\frac{1}{8} m \omega^{2} F_{0}{ }^{\prime \prime \prime}(\mathbf{L} \times \mathbf{v})^{2} \\
& -\frac{1}{16} \omega^{2} F_{0}{ }^{\prime \prime \prime} \nabla(\mathbf{L} \times \mathbf{r})^{2} \cdot \nabla U . \quad(\mathrm{A} 18
\end{aligned}
$$

Finally $F_{2}{ }^{C}$, the contribution arising from exchange, is readily obtained since the right-hand side of (A5) becomes

$$
\begin{aligned}
& -\frac{2 \pi e^{2}}{m^{2}} \frac{N-1}{N}\left[\mathbf{v} \cdot \nabla_{r} g_{0}-\frac{1}{m} \nabla_{r} U \cdot \nabla_{v} g_{0}\right] F_{0}{ }^{\prime} \\
& +\frac{2 \pi e^{2}}{m^{2}} \frac{N-1}{N} \omega\left[\mathbf{L} \times \mathbf{r} \cdot \nabla_{r} g_{0}-\mathbf{v} \times \mathbf{L} \cdot \nabla_{v} g_{0}\right] F_{0}{ }^{\prime} .
\end{aligned}
$$

It is not difficult to show that the second term of (A19) vanishes identically. Consequently, we have immediately

$$
F_{2}{ }^{C}=-\frac{2 \pi e^{2}}{m^{2}} \frac{N-1}{N} g_{0} F_{0}{ }^{\prime} .
$$

